\title{
Nanosurgical interventions in the practice of human ARTs
}

\author{
David F. Albertini
}

Published online: 17 June 2011

(C) Springer Science+Business Media, LLC 2011

Human ARTs have draw heavily upon observation and manipulations on a "micro" scale. Thanks in large measure to the evolution of the light microscope and digital imaging, the secrets of sperm, oocytes and embryos are no longer hidden from view but, rather, broadcast with aplomb throughout the embryology laboratory, on a daily basis. Current clinical practices offer patients an array of procedures that involve probing, injecting, sucking, and cutting our gametes and embryos - all under the skillful eyes of the embryologist and with the intent to achieve a pregnancy for our patients. Thus, ICSI, polar body or blastomere biopsy, assisted hatching et al, collectively reflect the translational incorporation of microscopy and micro scale engineering into the daily practices of human ARTs. So, how distant is the day when, as a matter of scale, ARTs enters the nanoworld?

Welcome to the world of the nanometer! While historically well within the purview of the student of ultrastructure as electron microscopists measured and described life (albeit fixed and embedded in plastic), the macromolecular dynamics of living cells have been exposed. Consider, for example, that the latest approaches for preimplantation genetic diagnosis bring to bear the nanometer resolution capabilities of CGH and other mapping techniques for the detection of gene dosage, mutations, translocations, or other ploidy aberrations associated with maternal, fetal or newborn health. As the complexities of our chromosomes continue to be revealed,

Capsule Prospects for improving oocyte quality by nanosurgical intervention are discussed in relation to recent studies involving transplanting nucleoli in mammalian eggs and embryos.

D. F. Albertini $(\bowtie)$

Kansas University Medical Center,

Kansas City, USA

e-mail: dalbertini@kumc.edu and the database that is our genome is deciphered at an alarming pace, the question becomes not whether, but when will nanosurgery take its place in the armament of ARTs?

Our lead article this month brings the prospects and concerns around nanosurgical interventions in human ARTs into perspective for our readership. The landmark studies of the Fulka, Jr. group, reported in Science in 2008 (Ogushi et al., Science 319:613-616), are notable for technological bravado and the dawning of a new era in micromanipulation of gametes and embryos. In addition to establishing the feasibility of removing and transplanting mammalian oocyte nucleoli into recipient zygotes, they were also able to show that nucleoli are maternally inherited and required for the embryo to generate its own supply of these vital organelles. Their work thus adds to the list of "essentialities" that presently includes our maternalized mitochondria. Accordingly, they posit the potential utility of nanosurgical organelle transplantation as a means towards improving embryo quality. The clinical basis for this idea stems from studies on human zygotes, showing that variations in the form, number, and alignment of nucleolar precursor bodies (NPBs, the progenitors of mature nucleoli) are predictive of embryo quality and pregnancy outcome.

It seems that, more often than not, we are hearing with increasing frequency that the quality of the embryo can be traced to one or another deficits harbored by the developing or maturing oocyte. Although implying that the oocyte is the raison d'etre for the embryo (excepting the confirmed parthenogeneticists among the readership) may be a stretch, the notion of maternal gamete conditioning has gained momentum, in recent years. And the prospect of improving or modifying egg quality by transplantation strategies is deeply rooted in the annals of embryology and cell biology.

For example, it is generally accepted that oocytes acquire developmentally important attributes that were 
lacking in substance or form, prior to maturation. The gain in function properties is acquired in response to the LH surge at ovulation in mammals. The first of these oocyte properties to be convincingly demonstrated derived from the classical cytoplasmic transfer experiments by the likes of Masui , Smith, and Markert. Eventually this work, in principle and design, set the stage for discovery of maturation promoting factor (MPF) by bypassing the progestin-induced signal that typically sets amphibian oocytes on their merry way through the rest of meiosis and on to a possible sperm encounter in the spirit of spring rituals.

While transferring cytoplasm between frog eggs hardly had the surgical aura about it that would elicit thoughts of therapeutic nanosurgery for human ARTs, these classical studies set into motion the identification of the factors that control the cell cycle. It is not in the too-distant past that most biologists and clinicians will recall efforts aimed at reversing the aging effect in oocytes by cytoplasmic transfer. These studies were designed to overcome the shortcomings of cytoplasm of an "older" egg in one patient by injecting it with cytoplasm that had been extracted from a younger oocyte donor. In retrospect, we accept the fact that our scientific understanding of attendant risks was too superficial to have appreciated or anticipated the detriments of mitochondrial heteroplasmy on offspring produced by this pace-setting procedure. But whether or not the crudeness of cytoplasm can be defined in absolute molecular terms, to address whatever the real problems with older eggs are, awaits future research. Some hints of what macromolecular assemblies have taken up shop in human oocytes that may be subject to transplantation are emerging.
Of this list of nano-objects, nucleoli, mitochondria, centrosomes, and perhaps other organelles, subserve an array of metabolic functions germane to embryogenesis. Fulka, Jr. and colleagues further point out that nucleoli hold promise for rehabilitating an older oocyte, given its multitasking persona. While long accorded the distinction of being the power-house for ribosome production (and thus a central player during oogenesis, to get this stockpile ready for the protein synthetic needs of the embryo), its true repertoire of jobs appears to be far more advanced and sophisticated than previously appreciated. For example, those cell-cycle factors mentioned earlier find asylum in the depths of the nucleolus, as it acts as a toll gate for ushering oocytes and somatic cells into and through specific stages of the cell cycle. Perhaps it is this role in cell-cycle regulation that underlies the striking tendency for human oocytes and embryos to fail at critical cell cycle decision points early in development.

Whatever may turn out to be the case, the strategy of supplementing oocyte cytoplasm is risky, in the absence of knowledge of composition and enzymology for the nucleolus or any other organelle one may have in mind. A thoughtful and critical approach that uncovers the link between oogenesis and embryogenesis is required to bring nanosurgical interventions into the everyday parlance of human ARTs. Call it rehabilitation, repurposing your oocytes, or a reconstruction project of nanometric proportions, offering nanoscale therapeutic options to our patients may someday require us to explain to our patients why their oocyte nucleoli have lost (or never acquired) the ability to support the embryo they so desperately seek. 